\title{
Decoupled Control for the Bicycling UGent Knee Rig: Design, Implementation, and Validation
}

\author{
Amélie Chevalier, Matthias Verstraete, Clara Ionescu, Member, IEEE, and Robin De Keyser
}

\begin{abstract}
This paper presents the design and implementation of a new control strategy for Ghent University, Ghent, Belgium (UGent) knee rig, which is capable of imposing bicycle motions on cadaver knee specimens in order to investigate knee biomechanics and the impact of newly designed knee implants. An electromechanical description of the system with its instrumentation and limitations is given. Via system identification, a dynamical model of the multipleinput/multiple-output system is obtained on which the control strategy design is based. This control strategy combines position control and force control. Dynamical analysis of the system suggests the need for a Proportional-IntegralDerivative (PID) control strategy with gain adaptation. In order to fulfill the performance specifications, a feed-forward action and decouplers are added to the control strategy, and their advantages are shown via simulations and experiments. The complete strategy is implemented on the real system and the output signals are measured for analysis. The results indicate that the identified model fits well to the measured data and that the designed control strategy is able to accurately control the system. The measurements show that the predefined performance specifications have been achieved for a bicycle motion with a period of $10 \mathrm{~s}$; the error on the position is smaller than $2 \mathbf{~ m m}$ and the error on the force is smaller than $10 \mathrm{~N}$.
\end{abstract}

Index Terms-Bicycle motion, control design, dynamic knee rig, feed-forward, gain adaptation, instrumentation.

\section{INTRODUCTION}

$\mathbf{T}$ HE KNEE joint is the most complex joint in the human body as it transfers large forces up to 3.5 times the body weight during the decent of a staircase [1]. Due to the complexity of the knee joint and the large loads transferred, the knee joint is keenly prone to injuries.

During the past decades, the number of knee joint injuries increased with a twofold reason. On one hand, statistical

Manuscript received November 23, 2016; revised February 10, 2017; accepted April 11, 2017. Date of publication April 24, 2017; date of current version August 14, 2017. Recommended by Technical Editor $\mathrm{H}$. A. Varol. The work of $\mathrm{M}$. Verstraete and $\mathrm{C}$. Ionescu was supported by the Flanders Research Center under Grant 12B3415N and Grant $12 N 5117 N$. (Corresponding author: Amélie Chevalier.)

A. Chevalier, C. Ionescu, and R. De Keyser are with the Dynamical Systems and Control Research Group, Department of Electrical Energy, Metals, Mechanical Constructions, and Systems, Ghent University, Ghent B-9052, Belgium (e-mail: amelie.chevalier@ugent.be; claramihaela.ionescu@ugent.be; robain.dekeyser@ugent.be).

M. Verstraete is with the Department of Physical Medicine and Orthopaedic Surgery, Ghent University Hospital De Pintelaan, Ghent B-9000, Belgium (e-mail: matthias.verstraete@ugent.be).

Color versions of one or more of the figures in this paper are available online at http://ieeexplore.ieee.org.

Digital Object Identifier 10.1109/TMECH.2017.2696708 analysis indicates that $37 \%$ of sport injuries are linked to the knee joint [2], [3]. On the other hand, due to the current aging population [4], [5], there is an increase in knee injuries due to wear and tear [6], [7].

For many patients with knee injuries, especially for elderly with osteoarthritis, the only possible treatment is total knee arthroplasty (TKA). During this procedure, the articulating surfaces of the knee joint are completely or partially replaced by artificial surfaces of a knee implant [8]. As a result, the amount of TKAs performed in EU and in U.S. has increased by 25-30\% during the past five years [9]-[11]. This increase coincides with a significant economic impact consisting of the cost of the procedure itself (9000 EUR or $\$ 15000$ USD) and the cost to the society due to the incapacity of the patient to work [10]-[12].

Although the design of knee implants and the surgical techniques have evolved tremendously [13], there is still a failure rate of $60.2 \%$ during the first five years after which a revision surgery is needed, increasing the economic impact even more [14], [15]. Besides infection, the two major reasons for failure are aseptic loosening of the components, accounting for $31.2 \%$ of all failures, and instability, accounting for $18.7 \%$ of all failures [14].

To evaluate the stability of new knee implant designs or new surgical techniques, dynamic knee rigs [16] are used in order to thoroughly understand the potential failure patterns and minimize the associated in vivo risks. These rigs are used by orthopedic researchers to impose natural movements to pre- and postoperative cadaver specimens.

The literature reports two types of dynamic knee rigs [17]. The first type applies forces and moments directly to the bones and is called a robotic knee system [18]. Here, the thigh bone is fixed on a pedestal while the shin bone is connected to the end-effector of a robotic arm applying different moments and forces. The second type applies an external force to the quadriceps muscle while leaving the knee kinematically free to find its equilibrium position. The advantage of the second type is that no constraints are imposed on the movement of the bones as is the case for the first type of machines [17] and that it is less expensive than the robotic knee systems.

The most common example of the second type is the Oxford knee rig (OKR), which is a simulator that imposes squat movements onto the knee joint by moving the hip joint vertically and applying a quadriceps force. The OKR is used as a basis for many knee rig designs [19]-[22] from which some have been extended in order to provide a wider range of 
motions. For example, the Purdue knee simulator [22] is able to simulating walking but with the disadvantage that the ankle is fixed. In order to provide a more general loading, a new type of knee rig has been developed by Forlani et al. which applies force and moments to the tibia using a cable structure [23], [24]. The downside is that the tibia always remains in the vertical direction limiting the flexibility.

Historically, a squat movement was selected as it can be seen as a model for a number of activities of daily living, such as rising from a chair [25]. However, this movement is limited in describing a number of patient-related issues with TKA today, e.g., instability during stair descent and bicycle motions. Therefore, Ghent University, Ghent, Belgium (UGent), knee rig (UGKR) has been developed, which has more flexibility in motion as the ankle joint is not fixed contrary to all previously discussed knee rig designs [26]. Cyclic motion patterns deserve particular attention as they are used in physical therapy after TKA [27], [28]. The benefit of the cycling motion is that it increases the range of motion of the postoperative knee while minimizing the stress on the joint [29]. Compared to the squat motion, which was selected in the previous literature, the load during a bicycle motion is lower. However, while the quadriceps force profile during the squat motion has a sinusoidal shape [30], the change in force during the bicycle motion is more sudden, thus more challenging for the control strategy. Even more sudden and higher changes in forces are observed during the descending staircase, which will be a subject of future work [31].

Using multiple actuators, the UGKR can impose a cyclic motion onto the knee joint but also be extended toward other clinically relevant motion patterns through the independent control of the kinematic and kinetic boundary conditions. Achieving repeatable motion patterns is of key importance in order to obtain clinically relevant data. Therefore, a suitable control strategy is needed to impose the positions and forces in the dynamic knee rig. The design of this control strategy and its validation is the subject of this research.

From literature studies on the control strategy of the OKR, two types of control strategies can be distinguished: force control and position control. A combination of both is often applied within the same simulator [22], [32], [33]. The Purdue knee simulator [22] is controlling five axes in order to impose squat motions, where the actuators can be controlled in either position or force mode. Controlling each of them with their own proportionalintegral-derivative (PID) controller does not provide satisfactory results due to the interaction between some of the axes. To deal with these cross couplings, Maletsky and Hillberry implemented a multiple-input-multiple-output (MIMO) control strategy [22].

In this paper, a model-based control strategy is developed for the UGKR based on an analysis of the cross couplings in the machine and its mechanical and electrical limitations. To evaluate the performance of the control strategy, the absolute error is used in the literature as performance metric [21], [23], [34]. For dynamic knee rigs, Forlani et al. report a position accuracy of 2.05-2.76 mm [23]. The accuracy on the force in dynamic knee simulators is reported between 10 and $30 \mathrm{~N}$ [21], [34]. The UGKR is combined with motion tracking devices in order to investigate the position of the bones and provide

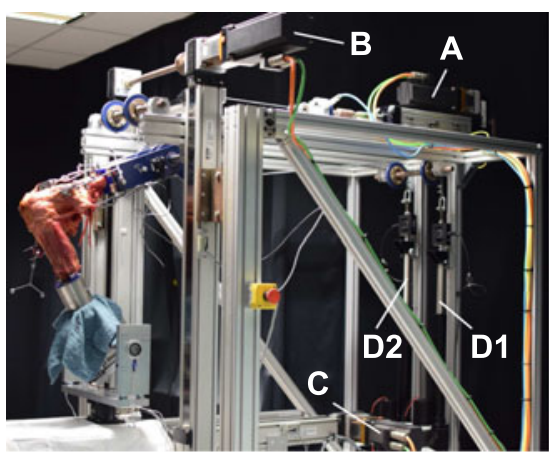

Fig. 1. UGKR mounted with a cadaver knee. The two position actuators are indicated by $\mathrm{B}$ and $\mathrm{C}$, while the three force actuators are indicated by A, D1, and D2.

a measure for instability of the knee. Therefore, accuracy on position and force is important as there is a strong link between them. Literature reports on statistically relevant differences in torques and forces due to small position changes [35], [36].

This paper is structured as follows. Section II gives a short description from electromechanical point of view of the UGKR and presents the used instrumentation and its limitations. Section III gives identification of the model for the model-based control design. Section IV presents the designed control strategy consisting of gain adaptation, PID control, feed-forward control, and decoupled control. Section V presents a set of simulations and experimental validation to investigate the performance of the designed controller. Discussion of the results is provided in Section VI. Conclusions and future work are presented in Section VII.

\section{UGENT KNEE RIG}

This section provides a description of the UGKR from electromechanical point of view combined with the used instrumentation and the limitations of the system. A detailed biomechanical description can be found in [26], where a basic ONOFFcontrol strategy was used resulting in a good but very slow performance of the system (one cycle per minute).

\section{A. System Description}

Contrary to the OKR, the hip joint is fixed in the UGKR and the ankle joint can move in the sagittal plane, i.e., the plane dividing the human body in left and right. Another difference with the OKR is that the UGKR not only controls the quadriceps force but also the hamstring forces. The latter have shown a considerable contribution to the knee kinematics [32].

The UGKR (see Fig. 1) consists of five actuators imposing motions and forces to the knee specimen. The position of the ankle joint is controlled in the sagittal plane ( $X Y$ plane) by actuators $\mathrm{B}$ and $\mathrm{C}$. Actuator $\mathrm{C}$ moves the ankle in horizontal or in the $X$-direction over a sliding platform. This entire platform together with the ankle and actuator $\mathrm{C}$ can move vertically, or in the $Y$-direction, by actuator B. While the movement of the ankle is imposed, the desired quadriceps force (i.e., front muscle in the upper thigh) and hamstring forces (i.e., back muscles 


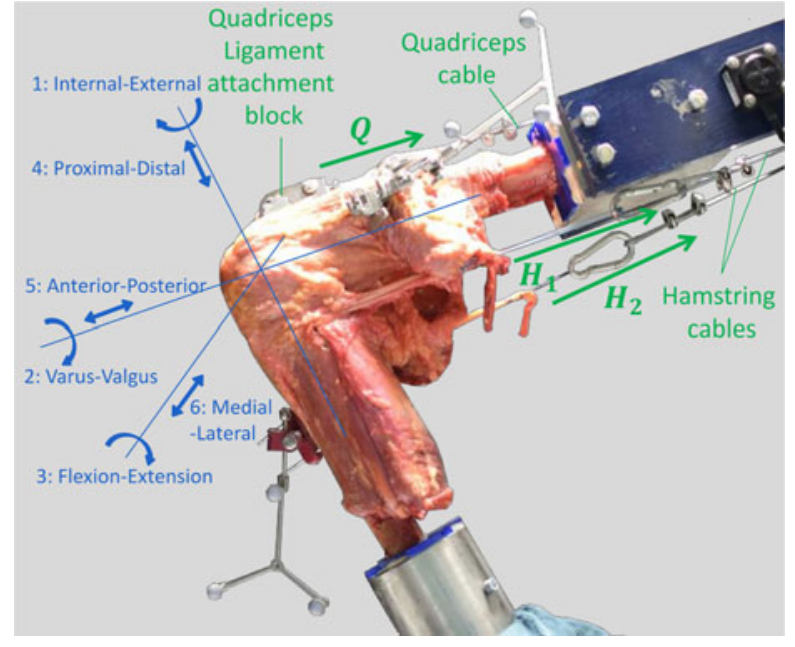

Fig. 2. Detailed view of the biological knee with its muscle connection mechanisms for the quadriceps and hamstrings and its natural six degrees of freedom.

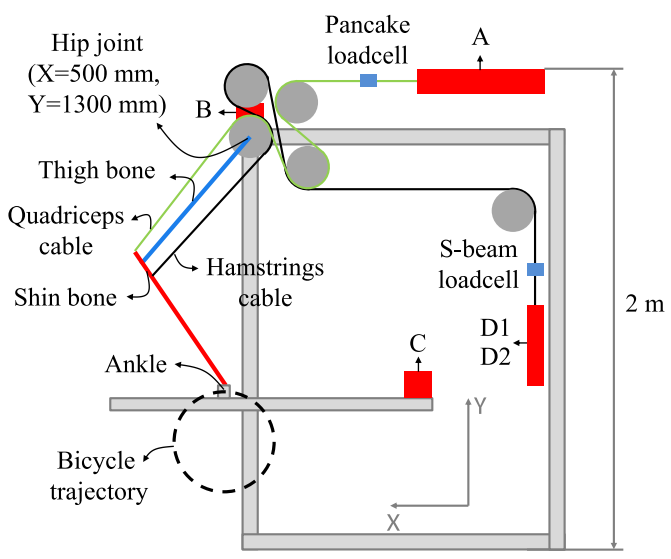

Fig. 3. Schematic representation of the UGKR.

in the upper thigh) are applied by actuator A and actuators D1 and D2, respectively. The hamstring force is mimicked by two actuators due to the different attachment sites of the anatomical hamstrings. Two attachment sites can be distinguished; one on the inside of the knee joint, i.e., medial, and one on the outside of the knee joint, i.e., lateral, creating a medial and lateral hamstring actuator. A detailed view of the biological knee with its muscle connections and its six natural degrees of freedom can be seen in Fig. 2. The applied forces are transferred to the corresponding tendons via a cable-pulley system. A schematic representation of the system can be seen in Fig. 3 .

Different types of specimens can be inserted in the system, such as a mechanical hinge that acts as a model for the human knee; a saw bone, i.e., a hard foam model of the knee joint; and a cadaveric knee joint.

In the presented research, the mechanical hinge model is used to test the designed control strategy, perform analyses, and validate the correct operation. The masses and lengths of the thigh and shin bone of the mechanical hinge are given in Table I.
TABLE I

PARAMETERS OF THE UGKR

\begin{tabular}{lc}
\hline \hline MECHANICAL HINGE & \\
\hline Length thigh bone & $421 \mathrm{~mm}$ \\
Length shin bone & $479 \mathrm{~mm}$ \\
Mass thigh bone & $11 \mathrm{~kg}$ \\
Mass shin bone & $5 \mathrm{~kg}$ \\
\hline POSITIONING ACTUATORS & Parker SMH Servo Motor \\
Maximal speed for the horizontal actuator & $174 \mathrm{~mm} / \mathrm{s}$ \\
Maximal speed for the vertical actuator & $122 \mathrm{~mm} / \mathrm{s}$ \\
\hline LINEAR POSITIONING SENSOR & Temposonics E-series \\
Horizontal range & $1000 \mathrm{~mm}$ \\
Vertical range & $1500 \mathrm{~mm}$ \\
Output signal & $0-10 \mathrm{~V}$ \\
Nonlinearity error & $<0.02 \%$ of the full range \\
Repeatability error & $<0.005 \%$ of the full range \\
Peak-to-peak disturbance & $1 \mathrm{~mm}$ \\
\hline LOAD CELLS & Sensy $2961 / \mathrm{S}$-beam 700 \\
Sensy range & $5 \mathrm{kN}$ \\
Sensy combined error & $<0.1 \%$ of the full range \\
Sensy peak-to-peak disturbance & $5 \mathrm{~N}$ \\
S-beam range & $2 \mathrm{kN}$ \\
S-beam combined error & $4 \mathrm{~N}$ \\
S-beam peak-to-peak disturbance & $<0.05 \%$ of the full range \\
\hline
\end{tabular}

\begin{tabular}{|c|c|}
\hline Manipulated & Controlled \\
\hline$V_{X} \quad \begin{array}{c}\text { voltage } \\
\text { horizontal }\end{array}$ & $\begin{array}{c}\text { horizontal } \\
\text { position }\end{array}$ \\
\hline $\begin{array}{ll}V_{Y} & \text { voltage } \\
\text { vertical }\end{array}$ & $\begin{array}{l}\text { vertical } \\
\text { position } Y\end{array}$ \\
\hline$V_{Q} \quad \begin{array}{c}\text { voltage } \\
\text { quadriceps }\end{array}$ & $\begin{array}{c}\text { quadriceps } \\
\text { force }\end{array}$ \\
\hline$V_{H 1 \text { hamstring } 1}$ & $\begin{array}{cc}\text { hamstring } \\
\text { force } 1\end{array} \mathrm{H}_{1}$ \\
\hline$V_{H 2 \text { hamstring 2 }}$ & $\begin{array}{c}\text { hamstring } \\
\text { force 2 }\end{array} \mathrm{H}_{2}$ \\
\hline
\end{tabular}

Fig. 4. Controlled and manipulated variables in the UGKR and their connections which show the direct paths and the cross interactions in this MIMO system.

Fig. 4 shows the controlled and manipulated variables in the UGKR together with the direct paths and the cross interactions of this MIMO system.

\section{B. Instrumentation and Limitations}

The knee rig is subjected to several limitations or constraints. These constraints originate first from the used actuators and sensors, which do not have an infinite range or accuracy. Properties such as nonlinearity, resolution, noise, and repeatability are limiting factors. Second, the limitation on the input currents of the servo motors and dc motors also gives a constraint as high accuracy combined with high speed will need a high input current. A third constraint is seen in the inner control loops of the servo drives. Although these drives respond very quickly, they are still subjected to the laws of physics and, therefore, have limitations on speed and accuracy.

1) Positioning Actuators: The positioning actuators, B and $\mathrm{C}$ in Fig. 1, are servomotors causing a linear displacement 
to the ankle joint due to an input voltage which represents the speed. They are manufactured by Parker and are equipped with an absolute rotary encoder used in the inner control loop, which will be treated as a black box model in this research. Each servomotor also has an individual Compax 3 control module, which processes the encoder data and controls the current, position, and velocity of the motor using LabVIEW. Due to the limitation on the input current, the speed of the actuators is limited (see Table I) and will determine the maximal angular velocity of the bicycle motion.

2) Force Actuators: Force actuator A in Fig. 1 is the same type of servomotor as actuator $\mathrm{B}$ and $\mathrm{C}$, where a force sensor, i.e., a pancake load cell, is placed between the cable and the piston to measure the applied quadriceps force. Force actuators D1 and D2 are dc motors that are linear ball actuators, which requires an input speed voltage and are controlled by an $\mathrm{H}-$ bridge. An external S-beam load cell is used to measure the hamstring forces.

3) Linear Sensors: Both positioning systems are equipped with linear position sensors, which are based on the time-based magnetostrictive position sensing principle [37]. Both sensors are E-series models manufactured by Tempsonics with characteristics listed in Table I. Both the nonlinearity and the repeatability limitations are negligible compared to the error caused by high-frequency noise on the analog voltage wires. The peakto-peak amplitude of the disturbances acting on the signal will affect the performance of a derivative action in the control strategy as this is sensitive to high-frequency signals. The output signals of the positioning sensors are transferred via a CompactRio module of National Instruments to the computer.

4) Load Cells: Three load cells are used by the UGKR: A pancake load cell, manufactured by Sensy, measures the quadriceps force and two S-beam load cells, manufactured by Load Cell Shop, are used to measure the hamstring forces. The load cell parameters are listed in Table I. The peak-to-peak amplitude of the disturbances for the Sensy sensor has the same order as the error amplitude making the error on the load cell itself no longer negligible compared to the noise. But as the high inertia of the actuator opposes the high-frequency control outputs, there will be only a small influence from the noise. The error caused by the load cell, on the other hand, cannot be accounted for. It is a fixed uncertainty and an important factor in terms of control. Trying to control the system within a margin of $1 \mathrm{~N}$ would be a waste of energy.

5) Control: Embedded control of the system is deployed using LabVIEW, which communicates via CompactRIO I/O modules of National Instruments. The LabVIEW program consists of an offline part that defines the reference trajectories and controller parameters, and an online part that maintains the realtime connection with the components and visualizes the sensor outputs.

\section{MOdEL IDENTIFICATION}

This section deals with the identification procedure and results of the entire UGKR in order to obtain a validated mathematical model describing the dynamics of the system when the mechanical hinge is inserted. The resulting models will be used to design the model-based control strategy. As the system is a MIMO system, both the direct paths and the cross interactions between the input and output variables of the system will be identified in order to investigate the significance of cross coupling (see Fig. 4). In the current research, a black-box approach is used in order to compare it in future work to a full mathematical model of the UGKR.

\section{A. Identification Procedure}

Identification is performed by applying a voltage pseudorandom binary signal (PRBS) as excitation signal at the input of one of the actuators and measuring the corresponding outputs, i.e., the positions or forces. During this measurement, the other four inputs are kept constant at the operating point, i.e., $100 \mathrm{~N}$ for the hamstrings, $350 \mathrm{~N}$ for the quadriceps, and varying for the $X$ - and the $Y$-position (see Table III). By performing the identification at normal operating conditions, the errors due to nonlinearity issues in the systems are reduced. This procedure is repeated for all five actuator inputs. A mathematical expression describing the dynamic behavior between the input and the output can then be obtained using a parametric identification technique called the prediction error method in MATLAB, which is expressed in simplified form as

$$
A\left(q^{-1}\right) z(t)=B\left(q^{-1}\right) v\left(t-n_{k}\right)+e(t)
$$

where $q^{-1}$ represents the digital shift operator, $z(t)$ is the measured output, $v(t)$ is the applied input voltage signal, $e(t)$ represent the small residuals, $n_{k}$ is the delay, and polynomials $A\left(q^{-1}\right)$ and $B\left(q^{-1}\right)$ are the denominator and numerator of the discrete transfer function, respectively. Equation (1) is also called the ARX-method where no noise is assumed to be present. As this system has a low noise level, this assumption does not cause significant modeling errors. Detailed information on identification techniques can be found in [38].

Note that (1) is a linear model. However, as the system dynamics are dependent on angles, i.e., sines and cosines, the real system is nonlinear. Therefore, modeling errors will be introduced by using this linear model. Furthermore, it will be shown that the use of adaptive gains will decrease the modeling errors due to nonlinearity.

This identification procedure is used to identify all the submodels in the UGKR. Each time the identification measurement is performed twice: one used for the identification and one used for validation of the obtained mathematical model.

\section{B. Identified Models and Validation}

For each subsystem, the aforementioned identification procedure is performed. For both the horizontal and vertical actuator, a PRBS with an amplitude of $2 \mathrm{~V}$, a sampling period of $30 \mathrm{~ms}$, and a minimum pulse width of $120 \mathrm{~ms}$ is applied. For the cross interaction between the horizontal position and the quadriceps force, the input PRBS signal $v_{X}$ and the corresponding measured output $Q_{\text {meas }}$ are plotted in Fig. 5 together with the simulated output $Q_{\operatorname{sim}}$ providing a validation of the identification. The resulting mathematical model is given in Table II with the 


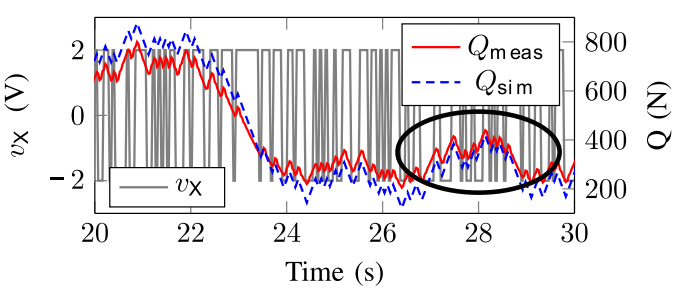

Fig. 5. Identification and validation for $G_{Q v_{X}}$. The input signal is the PRBS $v_{X}$, while the measured output signal is the force $Q_{\text {meas }}$. The resulting output of the identified system is indicated by $Q_{\mathrm{sim}}$. The identification is only valid in the region indicated by the ellipse.

TABLE II

IDENTIFIED TRANSFER FUNCTION MODELS

\begin{tabular}{lcc}
\hline \hline Model & Identification & NRMSE (\%) \\
\hline$G_{X v_{X}}$ & $\frac{0.496 z^{-3}}{1-z^{-1}}$ & $98.40 \%$ \\
$G_{Y v_{Y}}$ & $\frac{0.355 z^{-4}}{1-z^{-1}}$ & $98.06 \%$ \\
$G_{Q v_{Q}}$ & $\frac{15.830 z^{-2}}{1-z^{-1}}$ & $95.65 \%$ \\
$G_{Q v_{X}}$ & $\frac{-8.029 z^{-2}}{1-z^{-1}}$ & $91.58 \%$ \\
$G_{Q v_{Y}}$ & $\frac{1.993 z^{-2}}{1-z^{-1}}$ & $94.07 \%$ \\
$G_{H_{1} v_{H}}$ & $\frac{4.537 z^{-2}}{\left(1-z^{-1}\right)\left(1-0.800 z^{-1}\right)}$ & $95.02 \%$ \\
$G_{H_{1} v_{X}}$ & $\frac{4.490 z^{-2}}{1-z^{-1}}$ & $90.87 \%$ \\
$G_{H_{1} v_{Y}}$ & $\frac{0.957 z^{-2}}{1-z^{-1}}$ & $87.84 \%$ \\
$G_{H_{2}{ } H_{2}}$ & $\frac{3.380 z^{-2}}{\left(1-z^{-1}\right)\left(1-0.770 z^{-1}\right)}$ & $96.17 \%$ \\
$G_{H_{2} v_{X}}$ & $\frac{4.482 z^{-2}}{1-z^{-1}}$ & $88.65 \%$ \\
$G_{H_{2} v_{Y}}$ & $\frac{0.918 z^{-2}}{1-z^{-1}}$ & $88.66 \%$ \\
\hline \hline
\end{tabular}

corresponding normalized root-mean-squared error (NRMSE) in percentage, which is a measure of how well the response of the model fits the estimation data. Note that each obtained model consists of a pure integrator and minimal dynamics as expected from physical insight into the system as the actuators have a very fast inner control loop and the velocity in the system is integrated to obtain position.

Analogous, the models for the other subsystems can be obtained. For the identifications of the models linked to the quadriceps force $Q$, a PRBS with an amplitude of $2 \mathrm{~V}$, a sampling period of $30 \mathrm{~ms}$, and a minimum pulse width of $60 \mathrm{~ms}$ is used. For the identifications of the models linked to the hamstring forces $H_{1}$ and $H_{2}$, a PRBS with an amplitude of $1 \mathrm{~V}$, a sampling period of $30 \mathrm{~ms}$, and a minimum pulse width of $60 \mathrm{~ms}$ is used.

Note that tests have shown that only 11 out of the possible 25 interactions are present in the system as indicated in Fig. 4. The other interactions have, therefore, been omitted from the identification procedure.

\section{Control Strategy Design}

This section discusses each part of the designed control strategy for the UGKR. The performance specifications for the UGKR are set to an absolute position error less than $2 \mathrm{~mm}$ and an absolute force error less than $10 \mathrm{~N}$. These specifications are based on the reported literature on other knee rigs [21], [23], [34]. Note that these error limitations should be obtained
TABLE III

Gain Variations for the CROSS INTERACTIONS Where $X$ AND $Y$ ARE EXPRESSED IN MILLIMETER

\begin{tabular}{|c|c|c|c|c|c|c|}
\hline & \multicolumn{3}{|c|}{$G_{Q v_{X}}$} & \multicolumn{3}{|c|}{$G_{Q v_{Y}}$} \\
\hline & 700 & 500 & 300 & 700 & 500 & 300 \\
\hline 900 & -9.53 & -9.85 & -6.21 & 0.17 & 2.47 & 5.13 \\
\hline 750 & -7.77 & -7.17 & -4.59 & 1.92 & 3.25 & 5.00 \\
\hline 600 & -6.47 & -5.71 & -3.48 & 3.39 & 4.51 & 5.35 \\
\hline & \multicolumn{3}{|c|}{$G_{H_{1} v_{X}}$} & \multicolumn{3}{|c|}{$G_{H_{1} v_{Y}}$} \\
\hline & 700 & 500 & 300 & 700 & 500 & 300 \\
\hline 900 & 0.42 & 0.64 & 0.63 & 0.40 & 0.23 & 0.11 \\
\hline 750 & 0.44 & 0.55 & 0.59 & 0.36 & 0.37 & 0.01 \\
\hline 600 & 0.39 & 0.38 & 0.50 & 0.35 & 0.36 & 0.25 \\
\hline & \multicolumn{3}{|c|}{$G_{H_{2} v_{X}}$} & \multicolumn{3}{|c|}{$G_{H_{2} v_{Y}}$} \\
\hline$Y^{X}$ & 700 & 500 & 300 & 700 & 500 & 300 \\
\hline 900 & 0.36 & 0.51 & 0.45 & 0.35 & 0.19 & 0.08 \\
\hline 750 & 0.36 & 0.44 & 0.41 & 0.25 & 0.23 & 0.04 \\
\hline 600 & 0.24 & 0.37 & 0.39 & 0.25 & 0.32 & 0.16 \\
\hline
\end{tabular}

in regime, i.e., after all transients due to sudden steps in the reference signals have died out.

\section{A. Gain Adaptation}

When looking at the validation data from the identification for $G_{Q v_{X}}$ presented in Fig. 5, it can be noticed that the obtained model is only valid in a certain region (indicated in the ellipse). As the identification is performed at an initial quadriceps force of $400 \mathrm{~N}$, it can be concluded that the model is only valid around a certain operating point.

Therefore, the gain variation of the cross-interaction transfer functions is investigated by determining the static gain in several operating points. The resulting gains for each transfer function are shown in Table III.

The gain varies between 0.17 and 5.35 for $G_{Q v_{Y}}$, which indicates the nonlinearity of the model as noted in Section III. The effect of these gain variations is significant as the resulting output to an input between 0 and $10 \mathrm{~V}$ will be bigger than the inherent error of $5 \mathrm{~N}$ due to the instrumentation errors on the load cells discussed in Section II-B. In order to provide optimal control for the UGKR system, the controller should take the gain variations of the cross-coupling transfer functions into account. Therefore, an adaptive gain strategy is used for each of the six cross-coupling transfer functions where a linear interpolation is performed based on the gain variation matrices shown in Table III in order to obtain the intermediate gains. As linear interpolation is used to estimate a gain of a nonlinear system, modeling errors are introduced. These errors can be reduced by identifying the system's gains in more operating points but at the cost of an increased identification time. A tradeoff between identification and nonlinearity errors has been made resulting in an optimal of nine different operating points for identification in order to obtain the required control performance.

\section{B. Model-Based PID design}

An incremental PID controller is chosen to control the positions and forces in the UGKR. The incremental digital PID 
TABLE IV

TUNING SPECIFICATIONS AND PID PARAMETERS

\begin{tabular}{lcccccc}
\hline \hline Model & OS & $T_{\text {set }}$ & $R o$ & $K_{p}$ & $T_{i}$ & $T_{d}$ \\
\hline$G_{X x}$ & $<5 \%$ & $<1 \mathrm{~s}$ & $>0.55$ & 0.5 & 0.333 & 0 \\
$G_{Y y}$ & $<5 \%$ & $<1 \mathrm{~s}$ & $>0.55$ & 0.45 & 0.5 & 0 \\
$G_{Q q}$ & $<5 \%$ & $<0.5 \mathrm{~s}$ & $>0.55$ & 0.03 & 0.17 & 0 \\
$G_{H_{1} h_{1}}$ & $<5 \%$ & $<1 \mathrm{~s}$ & $>0.5$ & 0.016 & 0.38 & 0.079 \\
$G_{H_{2} h_{2}}$ & $<5 \%$ & $<1 \mathrm{~s}$ & $>0.5$ & 0.024 & 0.36 & 0.072 \\
\hline \hline
\end{tabular}

controller is expressed by

$$
u(t)=u(t-1)+c_{0} e(t)+c_{1} e(t-1)+c_{2} e(t-2)
$$

with

$$
\begin{aligned}
& c_{0}=K_{p}\left(1+\frac{T_{s}}{T_{i}}+\frac{T_{d}}{T_{s}}\right) \\
& c_{1}=-K_{p}\left(1+2 \frac{T_{d}}{T_{s}}\right) \\
& c_{2}=K_{p} \frac{T_{d}}{T_{s}}
\end{aligned}
$$

where $u$ is the controller output, $t$ is the current time sample, $e$ is the error signal (i.e., the difference between the current output and the desired output), $T_{s}$ is the controller sampling period, which is $30 \mathrm{~ms}$, and $K_{p}, T_{i}$, and $T_{d}$ are the tuning parameters of the controller.

The PID parameters are tuned using FRTool [39] and the mathematical models obtained in Section III-B. Specifications on overshoot OS, settling time $T_{\text {set }}$ and robustness $R o \subset[0,1]$ are used to find optimal controller parameters. The values for the tuning specifications along with the resulting PID parameters are given in Table IV. Notice that the given specification can be achieved by a PI controller for the $X$ - and $Y$-positioning and the quadriceps force. For the control of the hamstring forces, a PID controller is needed to fulfill the given specifications.

\section{Feed-Forward Control}

In order to improve the performance of the PID controllers, a feed-forward strategy is introduced. Simulations suggest that the PID controller on its own lacks performance in trajectory tracking (see Section V-A). Feed-forward uses a priori knowledge about the trajectory to estimate the future speed by performing a discrete differentiation on the future position taking into account for delay if present. This speed serves as a base control output on which the PID controller eliminates small errors. The feed-forward action then consists of a differentiation multiplied with a gain and can be expressed for the horizontal positioning as

$$
F_{X}=\frac{1-z^{-1}}{K T_{s}}
$$

where $K T_{s}$ is equal to the static gain of the identified model (see Table II) and $T_{s}$ is the sampling time of $30 \mathrm{~ms}$. Similar expressions for the other feed-forward controllers on the positions and the forces can be obtained.

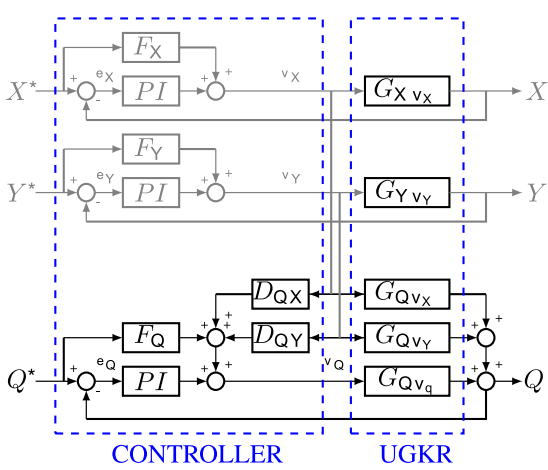

Fig. 6. Control diagram of the quadriceps control with the decouplers and the feed-forward control. A similar control scheme is used for each of the hamstrings.

\section{Decoupled Control}

Identification indicates that cross coupling between the forces and the positions is present. In order to optimally control the UGKR, the effect of the cross coupling will be compensated by the use of decouplers. The control scheme of the feed-forward PID control with the decouplers for the quadriceps force is given in Fig. 6. Here, $e_{i}$ for $i=X, Y, Q$ represents the difference between the desired value and the actual value and $v_{i}$ for $i=$ $X, Y, Q$ is the control effort, i.e., the process input.

The influence of the horizontal and vertical positioning on the quadriceps force is captured by transfer functions $G_{Q v_{X}}$ and $G_{Q v_{Y}}$. The decouplers $D_{Q X}$ and $D_{Q Y}$ should, thus, be constructed to counteract the cross-coupling effect. From Fig. 6, it can be directly observed that $D_{Q i} \cdot G_{Q v_{Q}}=-G_{Q v_{i}}$, for $i=$ $X, Y$, will result in decoupling, from which

$$
\begin{array}{r}
D_{Q i}=-\frac{G_{Q v_{i}}}{G_{Q v_{Q}}}=-\frac{\frac{K_{Q i} \cdot z^{-2}}{1-z^{-1}}}{\frac{K_{Q Q} \cdot z^{-2}}{1-z^{-1}}}=-\frac{K_{Q i}}{K_{Q Q}}=K_{D_{Q i}} \\
\text { for } i=X, Y .
\end{array}
$$

A similar reasoning can be applied to determine the decouplers for both hamstring control loops. The resulting expression is valid

$$
\begin{aligned}
D_{H_{1} X} & =-\frac{G_{H_{1} v_{X}}}{G_{H_{1} v_{H_{1}}}}=-\frac{\frac{K_{H_{1} X} \cdot z^{-2}}{1-z^{-1}}}{\frac{K_{H_{1} H_{1}} \cdot z^{-2}}{\left(1-z^{-1}\right)\left(1-0.8 z^{-1}\right)}} \\
& =-\frac{K_{H_{1} X}\left(1-0.8 z^{-1}\right)}{K_{H_{1} H_{1}}}=K_{D_{H_{1} X}}\left(1-0.8 z^{-1}\right) .
\end{aligned}
$$

For the other decouplers, similar expressions are obtained, resulting in

$$
\begin{aligned}
& D_{H_{1} Y}=K_{D_{H_{1} Y}}\left(1-0.8 z^{-1}\right) \\
& D_{H_{2} X}=K_{D_{H_{2} X}}\left(1-0.77 z^{-1}\right) \\
& D_{H_{2} Y}=K_{D_{H_{2} Y}}\left(1-0.77 z^{-1}\right) .
\end{aligned}
$$

Equations (5)-(7) are used to implement the decoupled control strategy. 


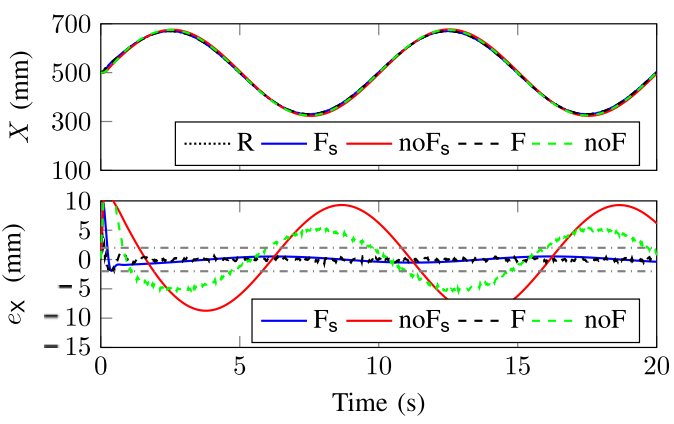

Fig. 7. Feed-forward control on the $X$-position with "R" indicating the reference signal, " $F$ " indicating with feed-forward action, and "noF" indicating without feed-forward action. The subscript " $s$ " indicates the simulated results. The dash-dotted line indicates the $2 \mathrm{~mm}$ position specification, which is not violated when feed-forward action is present.

\section{SimULATIONS AND EXPERIMENTAL VALIDATION}

The designed control strategy consists of a PID controller with gain adaptation where feed-forward action and decouplers are added to improve the performance. In this section, the advantages of the feed-forward and the decouplers are investigated using simulations in MATLAB/Simulink and experimental validation. The Simulink model consists of the transfer functions obtained from the identification in Section III-B. The full control strategy is implemented for a bicycle motion on the mechanical hinge.

\section{A. Performance of Feed-Forward Control}

To investigate the advantages of the feed-forward action, a discrete-time simulation with gain adaptation has been performed with and without feed-forward action. The same experiment is also performed on the real machine.

For the purpose of position control, the reference is a sinusoidal trajectory shown in the top of Fig. 7 for the $X$-position. The tracking performance with and without feed-forward action is evaluated using the absolute error between the reference and the actual output as defined in Fig. 6 and shown in Fig. 7.

A similar analysis can be performed for the quadriceps control where the reference is a force trajectory as seen during a bicycle motion. The bicycle motion starts with the upper leg at the top of the motion, i.e., at the moment in the bicycle motion where the biker needs to apply force. Afterward, the force decreases and remains constant when the other leg takes over. This reference signal is generated by a numerical model called AnyBody [26]. The resulting output signals and error signals for both with and without feed-forward action are shown in Fig. 8.

\section{B. Performance of Decoupled Control}

To investigate the performance of the decouplers, a set of step changes has been designed where each input variable alternately receives a stepwise change as can be seen from the dashed reference signals in Fig. 9. As these stepwise changes are very abrupt, this experiment is only performed in simulation due to safety of the mechanical hinge. From the output signals in Fig. 9, it is clear that interactions between the forces and positions are
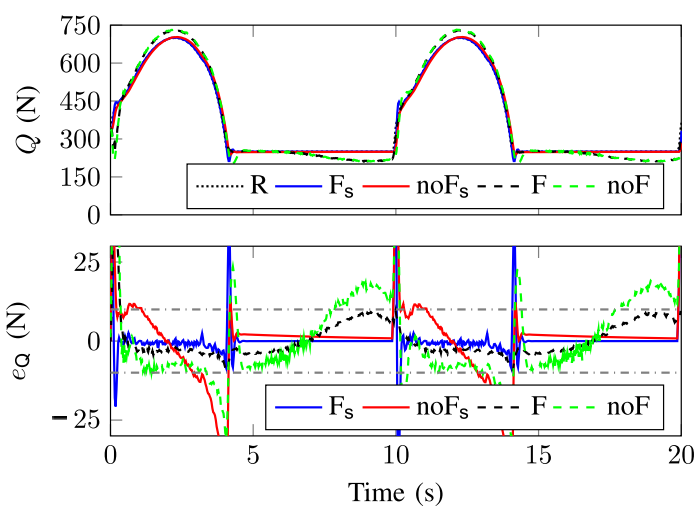

Fig. 8. Feed-forward control on the $Q$-force with "R" indicating the reference signal, " $F$ " indicating with feed-forward action, and "noF" indicating without feed-forward action. The subscript " $s$ " indicates the simulated results. The dash-dotted line indicates the $10 \mathrm{~N}$ force specification which is in regime not violated when feed-forward action is present.
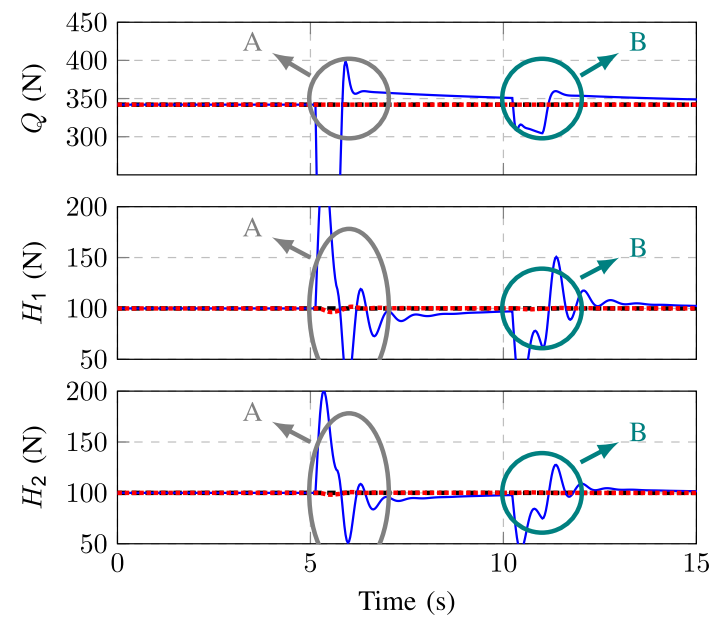

Fig. 9. Decoupled versus nondecoupled control (black dashed: reference signal, blue solid: simulated output without decouplers, red dotted: decoupled simulated output). Event $A$ is a step change on the $X$-position, while event B is a step change on the $Y$-position. The effect of both events is fully compensated by the decouplers.

present. A step on the horizontal positioning (event A: step from 500 to $600 \mathrm{~mm}$ ) has a significant effect on the quadriceps and hamstring forces (see gray circles). The same is valid for the vertical positioning (see green circles, event B: step from 850 to $750 \mathrm{~mm})$.

\section{Performance of the Proposed Control Strategy}

The designed control strategy is applied on a mechanical hinge, which serves as a model for the human knee joint [26]. The performed motion are bicycle motions with a cycling period of $10 \mathrm{~s}$. The reference signals for the horizontal and vertical positioning are sinusoids, the reference for the quadriceps force is the obtained signal from AnyBody, and for the hamstring forces a constant value of $100 \mathrm{~N}$ is considered. The output values are measured and compared to the simulated values in order to validate the obtained model from identification. A close up of the results is shown in Fig. 10. 

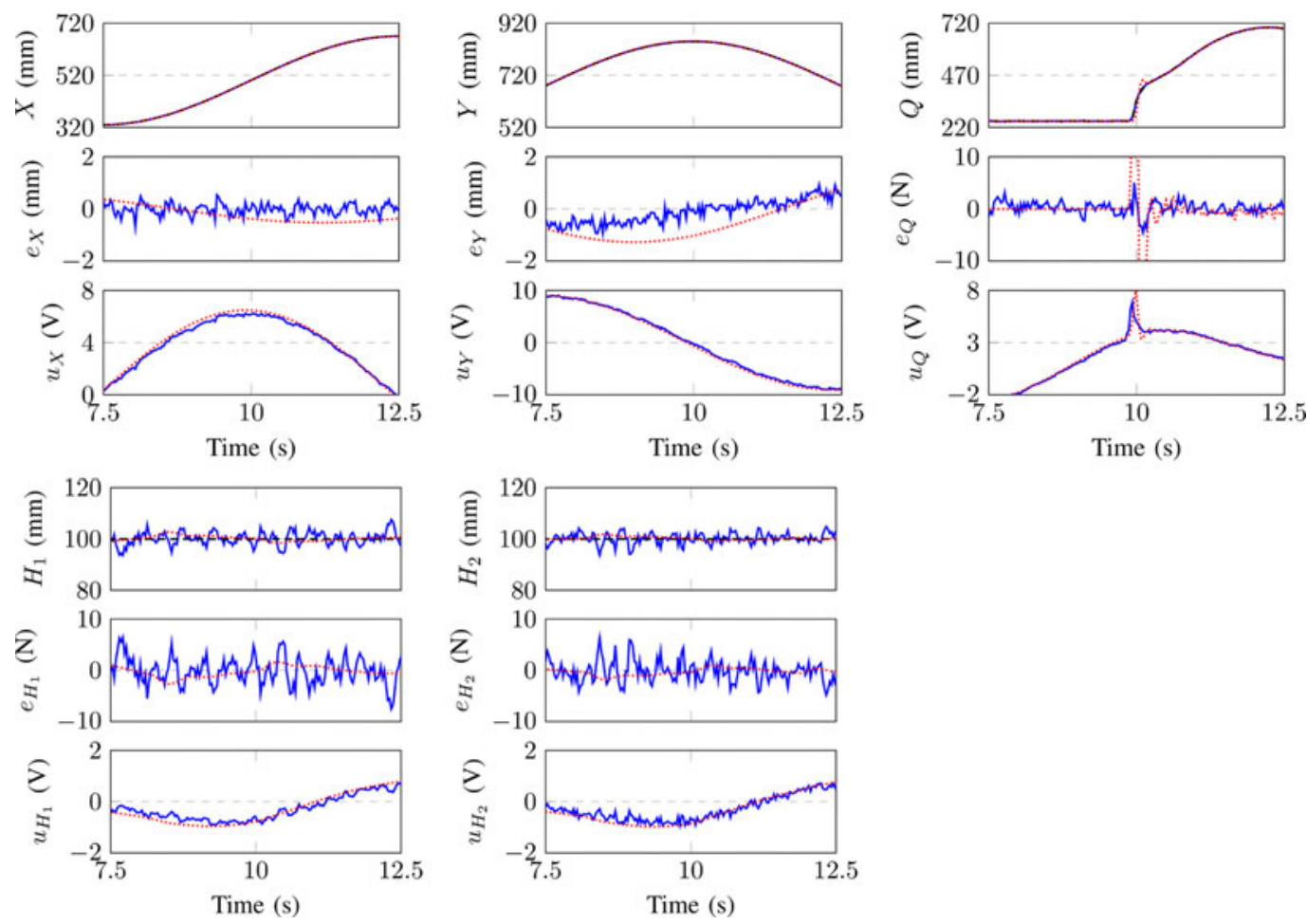

Fig. 10. Measured signals (solid blue line) compared to the simulated signals (dotted red line) to show how good the correspondence is between the measured signals and the simulated signals based on the identification. The reference signals are plotted in dashed black lines.

\section{DISCUSSION}

The simulations and experimental results from Section $\mathrm{V}$ require some discussion.

The results on the performance of the feed-forward control suggest that using the feed-forward controller leads to a decrease in the absolute error between the desired position or force and the actual output. For the position, maximal error after transient behavior decreases from 5.5 to $0.6 \mathrm{~mm}$ by using feed-forward in the real measurement. This decrease in error leads to a satisfaction of the position design specification of maximal $2 \mathrm{~mm}$ absolute error for both the simulation as the real measurements. This limit is indicated by the dash-dotted line in Fig. 7. For the force, a similar decrease is observed in Fig. 8, where the error decreases from a maximal error after transient behavior of 19.2 to $9.7 \mathrm{~N}$ for the real measurement. The error is decreased below $10 \mathrm{~N}$, i.e., the design specification, at the cost of a slightly higher control effort. However, the control effort remains within the $\pm 10 \mathrm{~V}$ range imposed by the limitations of the instrumentation as discussed in Section II-B.

The results on the performance of the decouplers in Fig. 9 indicate that the designed decouplers compensate the crossinteraction effect completely for a step change on the $X$-position (event A) and the $Y$-position (event B).

The comparison between the response of the simulated model to the one of the real system in Fig. 10 motivates the validation of the identified model. From Fig. 10, it can be concluded that the designed control strategy is able to control the system within the given design parameters, i.e., less than $2 \mathrm{~mm}$ error on positioning and less than $10 \mathrm{~N}$ error on force, which are comparable to knee rigs from the literature. From the measurements, it can be concluded that this system is able to perform a bicycle motion at a cycling period of $10 \mathrm{~s}$, which is six times faster than the previous version. This version of the UGKR is to the authors' knowledge the first dynamic knee rig able to impose bicycle motions with high accuracy and speed.

The experimental results show that the identified gains used in the gain adaptation techniques are adequate for the mechanical hinge. However, as the difference in elasticity and nonlinearity between the mechanical hinge and the cadaver knee is big, the identified gains will not suffice when a cadaver specimen is inserted in the UGKR. Therefore, an automatic gain identification sequence will need to be added to the proposed system in order to cope with this problem as part of the future work. In this way, the sequence can be run when a new cadaver specimen is inserted, thus providing a solution for the interpatient variability in the gains.

Notice the sudden changes in the quadriceps force reference trajectory needed to simulate the bicycle motion. Even though the amplitude of the force is lower compared to simulated squat motions, the reference trajectory is more challenging from the control point of view than the sinusoidal force profiles seen during squat motions. This bicycle motion is a starting point to simulate suddenly changing force trajectories such as the descending staircase which will be focus of future work.

\section{CONCLUSION}

This paper presents a new control strategy for the UGent knee rig, which is capable of imposing bicycle motions onto cadaver 
knee specimens in order to analyze knee biomechanics and newly designed knee implants. The system is described from electromechanical point of view and its instrumentation and limitations are discussed. A new control strategy is designed based on a mathematical model obtained with identification techniques. Dynamical analysis suggests that there is a need for a PID controller with gain adaptation. In order to improve the control performance, feed-forward action and decouplers are added to the control strategy and their beneficial effect is shown via simulation and measurement. The final control strategy is implemented on the real system and tested using a mechanical hinge model of the knee joint. The results indicate that the obtained model fits well to the measured data and that the designed control strategy is able to control the system within the given specifications of less than $2 \mathrm{~mm}$ error on position and less than $10 \mathrm{~N}$ error on force. Future work includes testing the designed control strategy on a series of cadaver knees in order to investigate the effect of a biological specimen and test the robustness of the controller against specimen variability. Development of a mathematical model and its validation to the real system is a second part of the future work which can also be used to validate the clinical relevance of the $10 \mathrm{~s}$ cyclic motion.

\section{REFERENCES}

[1] I. Kutzner et al.,"Loading of the knee joint during activities of daily living measured in vivo in five subjects," J. Biomech., vol. 43, no. 11, pp. 21642173, Aug. 2010.

[2] M. Majewski, "Epidemiology of athletic knee injuries: A 10-year study," Knee, vol. 13, no. 3, pp. 184-188, Jun. 2006.

[3] M. De Loës, L. J. Dahlstedt, and R. Thomée, "A 7-year study on risks and costs of knee injuries in male and female youth participants in 12 sports," Scand. J. Med. Sci. Sports, vol. 10, no. 2, pp. 90-97, Apr. 2000.

[4] The Organisation for Economic Co-Operation and Development, "Ageing populations: High time for action," Apr. 2014. [Online]. Available: http://www.oecd.org/employment/emp/34600619.pdf

[5] G. Lanzieri, "The greying of the baby boomers: A century-long view of ageing in European populations," Eurostat Statist. Focus, Pop. Soc. Conditions, pp. 1-11, 2011.

[6] D. T. Felson, A. Naimark, J. Anderson, L. Kazis, W. Castellu, and R. F. Meenan, "The prevalence of knee osteoarthritis in the elderly. The Framingham Osteoarthritis Study," Arthritis Rheumatism, vol. 30, pp. 914-918, Aug. 1987

[7] G. Peat, R. McCarney, and P. Croft, "Knee pain and osteoarthritis in older adults: A review of community burden and current use of primary health care," Ann. Rheumatic Dis., vol. 60, pp. 91-97, Feb. 2001.

[8] C. S. Ranawat, "History of total knee replacement," J. Southern Ortho. Assoc., vol. 11, no. 4, pp. 218-226, 2002

[9] The Organisation for Economic Co-Operation and Development, "Health at a glance: Europe 2012," Aug. 30, 2013. [Online]. Available: http://www. oecd-ilibrary.org

[10] "FastStats: Inpatient surgery," Centers Dis. Control, Nat. Center Health Statist., Mar. 30, 2016. [Online]. Available: http://www.cdc.gov/nchs/ fastats/inpatient-surgery.htm

[11] S. Kurtz, F. Mowat, K. Ong, N. Chan, E. Lau, and M. Halpern, "Prevalence of primary and revision total hip and knee arthroplasty in the united states from 1990 through 2002," J. Bone Joint Surg. Amer., vol. 87, no. 7, pp. 1487-1497, Jul. 2005.

[12] Surgery Price, "Knee replacement price," Aug. 30, 2013. [Online]. Available: http://www.surgeryprice.co.uk

[13] E. K. Song, J. K. Seon, J. Y. Moon, and Y. Ji-Hyoun, "The evolution of modern total knee protheses," in Arthroplasty-Update, P. Kinov, Ed. Rijeka, Croatia: InTech, 2013, 10.5772/54343. [Online]. Available: http://www.intechopen.com/books/arthroplasty-update/the-evolution-ofmodern-total-knee-prostheses

[14] A. V. Lombardi, Jr., K. R. Berend, and J. B. Adams, "Why knee replacements fail in 2013: Patient, surgeon, or implant?" Bone Joint J., vol. 96, no. 11, pp. 101-104, Nov. 2014.
[15] K. G. Vince, "The problem total knee replacement: Systematic, comprehensive and efficient evaluation," Bone Joint J., vol. 96, no. 11, pp. 105-111, Nov. 2014.

[16] J. A. Shaw and D. G. Murray, "Knee joint simulator," Clin. Ortho. Related Res., vol. 94, pp. 15-23, Jul./Aug. 1973.

[17] P. S. Walker et al.,"A knee simulating machine for performance evaluation of total knee replacements," J. Biomech., vol. 30, no. 1, pp. 83-89, Jan. 1997.

[18] G. Li et al.,"Biomechanics of posterior-substituting total knee arthroplasty: An in vitro study," Clin. Ortho. Related Res., vol. 404, no. 1, pp. 214-225, Nov. 2002.

[19] J. P. Halloran, C. W. CLary, L. P. Maletsky, A. J. Petrella, and P. J. Rullkoetter, "Verification of predicted knee replacement kinematics during simulated gait in the Kansas knee simulator," J. Biomech. Eng., vol. 132, no. 8, pp. 1-6, Aug. 2010.

[20] M. Wünschel, O. Müller; J. Lo, C. Obloh, and N. Wülker, "The anterior cruciate ligament provides resistance to externally applied anterior tibial force but not to internal rotational torque during simulated weight-bearing flexion," Arthroscopy, vol. 26, no. 11, pp. 1520-1527, Nov. 2010.

[21] T. M. Guess and L. P. Maletsky, "Computational modeling of a dynamic knee simulator for reproduction of knee loading," J. Biomech. Eng., vol. 127, no. 7, pp. 1216-1221, Dec. 2005.

[22] L. P. Maletsky and B. M. Hillberry, "Simulating dynamic activities using a five-axis knee simulator," J. Biomech. Eng., vol. 127, pp. 123-133, Feb. 2005 .

[23] M. Forlani, N. Sancisi, M. Conconi, and V. Parenti-Castelli, "A new test rig for static and dynamic evaluation of knee motion based on a cable-driven parallel manipulator loading system," Meccanica, vol. 51, pp. 1571-1581, Jul. 2016.

[24] N. Sancisi, M. Conconi, M. Forlani, and V. Parenti-Castelli, "A test rig for the analysis of the knee under dynamic motion tasks," J. Med. Dev. vol. 9, no. 2, pp. 1-3, Apr. 2015, Art. no. 020934.

[25] A. B. Zavatsky, "A kinematic-freedom analysis of a flexed-knee-stance testing rig," J. Biomech., vol. 30, no. 3, pp. 277-280, Mar. 1997.

[26] M. Verstraete and J. Victor, "Possibilities and limitations of novel in-vitro knee simulator," J. Biomech., vol. 42, no. 12, pp. 3377-3382, Sep. 2015.

[27] N. Artz, K. T. Elvers, C. M. Lowe, C. Sackley, P. Jepson, and A. D. Beswick, "Effectiveness of physiotherapy exercise following total knee replacement: Systematic review and meta-analysis," BMC Musculoskeletal Disorders, vol. 16, pp. 1-21, Feb. 2015.

[28] M. J. Bade and J. E. Stevens-Lapsley, "Early high-intensity rehabilitation following total knee arthroplasty improves outcomes," J. Ortho. Sports Phys. Therapy, vol. 41, no. 12, pp. 932-941, Dec. 2011.

[29] T. E. Johnston, "Biomechanical considerations for cycling interventions in rehabilitation," Phys. Therapy, vol. 87, no. 9, pp. 1243-1252, Sep. 2007.

[30] R. F. Escamilla et al.,"Effects of technique variations on knee biomechanics during the squat and leg press," Med. Sci. Sports Exercise, vol. 33, no. 9, pp. 1552-1566, Sep. 2001.

[31] L. A. Fok, A. G. Schache, K. M. Crossley, Y. C. Lin, and M. G. Pandy, "Patellofemoral joint loading during stair ambulation in people with patellofemoral osteoarthritis," Arthritis Rheumatism, vol. 65, no. 8, pp. 2059-2069, Aug. 2013.

[32] J. Victor, L. Labey, P. Wong, B. Innocenti, and J. Bellemans, "The influence of muscle load on tibiofemoral knee kinematics," J. Ortho. Res. vol. 28, no. 4, pp. 419-428, Apr. 2010.

[33] L. Kirsch, C. J. Wirth, D. Kohn, and A. Glowik, "A dynamic knee simulator with feedback control," J. Biomech., vol. 31, no. 1001, p. 143, 1998.

[34] C. W. Colwell, Jr. and D. D. D'Lima, "The electronic knee," in Total Knee Arthroplasty. Berlin, Germany: Springer-Verlag, 2005, pp. 282-287.

[35] R. P. Hirata and M. Duarte, "Effect of relative knee position on internal mechanical loading during squatting," Revista Brasileira de Fisioterapia, vol. 11, no. 2, pp. 107-111, Mar 2007.

[36] A. C. Fry, J. C. Smith, and B. K. Schilling, "Effect of knee position on hip and knee torques during the barbell squat," J. Strength Cond. Res., vol 17, no. 4, pp. 629-633, Nov. 2003.

[37] C. Deng, Y. Kang, E. Li, Y. Zhang, J. Cheng, and T. Ge, "A new model of the signal generation mechanism on magnetostrictive position sensor," Measurement, vol. 47, pp. 591-597, Jan. 2014.

[38] A. Chevalier, J. A. Hernandez, C. M. Ionescu, M. Verstraete, and R. De Keyser, "Closed-loop identification of a multivariable dynamic knee rig," in Proc. IFAC Symp. Syst. Identification, Beijing, China, Oct. 2015, pp. $116-121$

[39] R. De Keyser and C. M. Ionescu, "FRtool: A frequency response tool for CACSD in Matlab," in Proc. IEEE Conf. Comput.-Aided Control Syst. Des., Munich, Germany, Oct. 2006, pp. 2275-2280. 


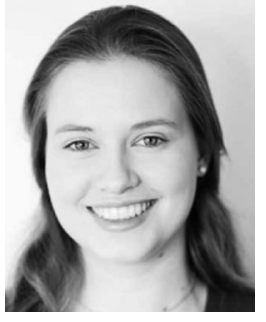

Amélie Chevalier was born in 1988, Ghent, Belgium. She received the M.Sc. degree in biomedical engineering from Ghent University, Ghent, in 2012.

She is currently a Ph.D. Researcher with the Department of Electrical Energy, Systems, and Automation, Ghent University. Her research interest focuses on control of industrial and biological systems, with a main focus on the biomechanics of the knee joint. She combines her research with a position of Teaching Assistant with Ghent University, where she focuses on educational tools for control engineering students.

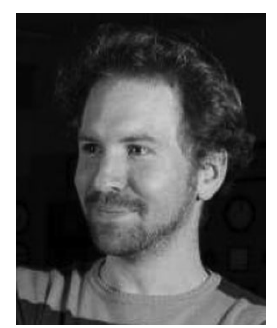

Matthias Verstraete was born in 1986 in Ghent, Belgium. He received the M.Sc. degree in mechanical engineering and the Ph.D. degree in fracture mechanics from Ghent University, Ghent, in 2009 and 2013, respectively.

Since 2014, he has been with the Department of Orthopaedic Surgery, Ghent University, focusing on orthopedic biomechanics. His work deals with the impact of total knee implants on the stability and kinematics of the knee.

Dr. Verstraete holds a prestigious postdoctoral grant from the Flanders Research Center, Brussels, Belgium, from 2016 onwards.

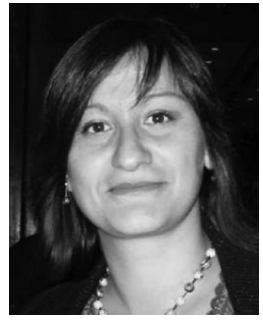

Clara lonescu (M'07) was born in 1979, Cimpulung, Romania. She received the M.Sc. degree in industrial informatics and automation from Dunarea de Jos University, Galati, Romania, in 2003, and the Ph.D. degree in biomedical engineering from Ghent University, Gent, Belgium, in 2009.

She is currently a Professor with Ghent University, involved in several international projects with both industrial and biomedical applications in identification and control.

Prof. Ionescu was the holder of a prestigious postdoctoral grant from the Flanders Research Center during 2011-2016.

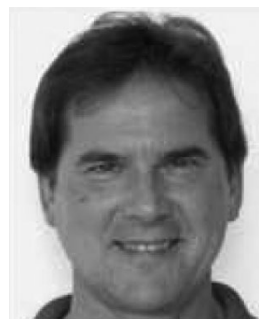

Robin De Keyser received the M.Sc. degree in electromechanical engineering and the Ph.D. degree in control engineering from Ghent University, Ghent, Belgium, in 1974 and 1980, respectively.

He is currently a Full Professor of control engineering with the Faculty of Engineering, Ghent University. $\mathrm{He}$ is an author/co-author of approximatley 300 publications in journals, books, and conference proceedings. He acted as an External Review Expert for several European Commission research programs and is one of the pioneers who produced the original concepts of predictive control during the 1980s. His teaching and research activities include model predictive control, autotuning and adaptive control, modeling and simulation, and system identification. His research interests include applications driven with many pilot implementations in technical and nontechnical systems, including chemical, steel, marine, mechatronic, semiconductor, power electronics, and biomedical systems. 